CASE REPORT

A.U. Kalnins

B. Geryk

W. Olivero

T.A. Kim

\title{
Spinal Epidural Lubricant Grease Collection Mimicking Traumatic Spinal Epidural Hematoma
}

SUMMARY: We report an unusual case of a patient with an epidural fluid collection with signal characteristics mimicking an epidural hematoma. The patient presented with myelopathy caused by thoracic spinal cord compression after a traumatic injury to the chest and back. The injury was caused by high-pressure injection of industrial-grade lubricant grease. This case demonstrates that cord compression can be caused by exogenous material in the setting of trauma that can mimic an acute epidural hematoma.

$\mathbf{T}$ he evolution of the MR imaging signal intensity characteristics of a spinal epidural hematoma is thought to follow the MR imaging characteristics of a hemorrhage in the brain. $^{1,2}$ This case demonstrates an unusual occurrence in which exogenous fluid mimicked the clinical and radiologic manifestations of early subacute traumatic spinal epidural hemorrhage.

\section{Case Report}

The patient was a 31-year-old man who sustained a traumatic injury to the right neck and right chest wall while trying to repair a pneumatic device on a hydraulic injection instrument of a bulldozer. The patient was struck in the neck with a "metal plug," resulting in a puncture of the right anterior neck and right upper chest wall. The patient was airlifted to a trauma center, and on arrival, he was noted to be hemodynamically stable and was ambulatory. The initial neurologic examination by the trauma service in the emergency department was reported to be normal. The patient was taken to the operating room for local exploration of the right neck wound, and a right thoracostomy was completed for drainage of the fluid, presumed to be a hemopneumothorax. A neck CT angiographic examination was done, which showed general soft tissue injury involving the chest wall and posterior paraspinous muscles, a small residual left pneumothorax, and hypoattenuated epidural fluid collection.

On day 2 of admission, a Foley catheter was inserted because of the patient's inability to void. During morning rounds on day 4 of admission, the patient exhibited new neurologic deficits with bilateral leg numbness, tingling, and motor deficits, with the right worse than the left. Neurosurgical consultation was obtained, along with spine CT and MR imaging.

MR imaging of the thoracic spine revealed a posterior epidural collection, which was interpreted as an epidural hematoma, extending from the T3 to the T6 level with some residual collection extending into the lower thoracic and upper lumbar spinal canal (Fig 1).

Received March 31, 2009; accepted April 7.

From the Department of Radiology (A.U.K.) and Neurosurgery Residency Program (B.G.), University of North Carolina, Chapel Hill, NC; and Departments of Neurosciences and Neurosurgery (W.O.) and Radiology (T.A.K.), Carle Clinic Association, Carle Clinic Radiology, Urbana, III

Previously presented at: Annual Meeting of the American Society of Neuroradiology and Neuroradiology Education and Research Foundation Symposium, May 31-June 5, 2008; New Orleans, La.

Please address correspondence to Thomas A. Kim, MD, Neuroradiology, Department of Radiology, Carle Clinic Association, 602 W University Ave, Carle Clinic Radiology, Urbana, IL 61801; e-mail: Thomas.Kim@carle.com

DOI 10.3174/ajnr.A1670
The patient was emergently taken to the operating room, and a decompressive laminectomy was performed from the T2 to the T6 level. There was a large collection of what was presumed to be pus and possibly old blood within the right paraspinous muscles at approximately T2 through T4. Once the ligamentum flavum was opened up, immediately more apparent dark epidural fluid was encountered, which was thought to represent a collection of pus and old blood. This fluid was evacuated and sent for culture. Initially thought to be pus, the viscous fluid was shown to represent industrial grease with some inflammatory cells. The same thick viscous fluid was evacuated from the epidural space and from within the muscles of the right upper back.

Additional investigation revealed that the patient had sustained a high-pressure injection of lubricant grease from the hydraulic device of a bulldozer, with pressures exceeding 3000 psi. The lubricant grease injection was postulated to have passed through the apex of the lung, potentially the mediastinal space, and into the posterior thorax, ending in the thoracic epidural space.

The lubricant grease was identified as Conoco Phillips Dynalife GC-LB, an automotive lithium complex grease. ${ }^{3}$ This lubricant grease was placed along with normal saline in a glass jar and imaged with the same scanner with the same T1 and T2 acquisition parameters. The acquired images demonstrate similar MR imaging signal intensity characteristics of the epidural fluid collection: hyperintensity on T1weighted imaging and hypointensity on T2-weighted imaging, with normal expected hypointensity and hyperintensity of the saline, respectively (Fig 2). These signal intensity characteristics mimic those of an early subacute epidural hemorrhage ( $>3$ days). ${ }^{1}$

\section{Discussion}

Traumatic spinal epidural hematomas (TSEHs) are relatively uncommon, occurring in less than $2 \%$ of all spinal injuries, but their sequelae, including cord compression, can be serious, which makes early diagnosis essential. ${ }^{4}$ Imaging plays a crucial role in this diagnosis, and MR imaging is the best tool for evaluation of a potential TSEH.

MR imaging of an early subacute spinal epidural hematoma displays a heterogeneously high signal intensity on T1weighted imaging and a heterogeneously low signal intensity on T2-weighted imaging. ${ }^{1,2}$ In the case of our patient, the presumed acute spinal epidural hematoma had similar T1 and T2 signal intensity characteristics.

CT imaging of a spinal epidural hematoma displays a high-attenuation collection within the spinal canal. ${ }^{1}$ From a retrospective standpoint, the intraspinal epidural collection reported on CT angiography of the neck in this patient re- 

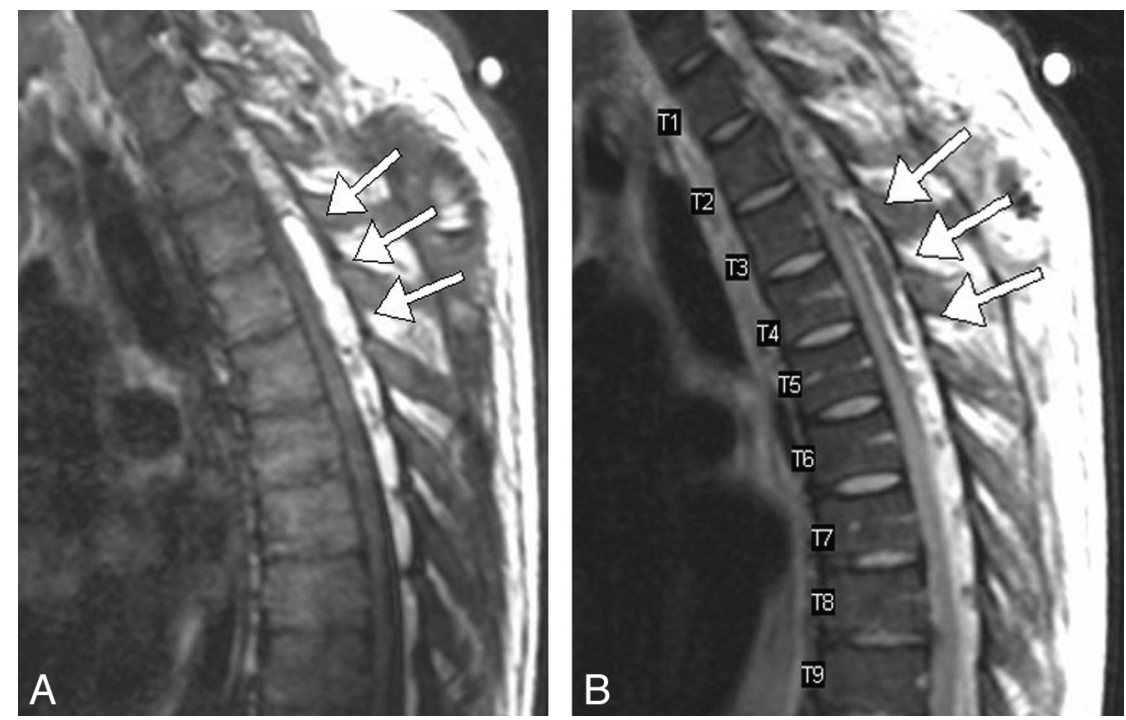

Fig 1. Sagittal T1 and T2 turbo-spin-echo sequences of the posterior spinal canal. $A$, Sagittal $\mathrm{T} 1$ spin-echo sequence revealing mixed signal intensity in the posterior spinal canal most marked from approximately the T1 level to the $\mathrm{T} 7$ level, with minimal findings extending into the lower thoracic and upper lumbar levels. There is an area of high signal intensity extending from the T3 to the T5 level (arrows). B, Sagittal T2 turbo-spin-echo sequence revealing mixed signal intensity in the posterior spinal canal most marked from approximately the T1 level to the $\mathrm{T} 7$ level, with minimal findings extending into the lower thoracic and upper lumbar levels. There is an area of low signal intensity extending from the T3 to the T5 level (arrows).
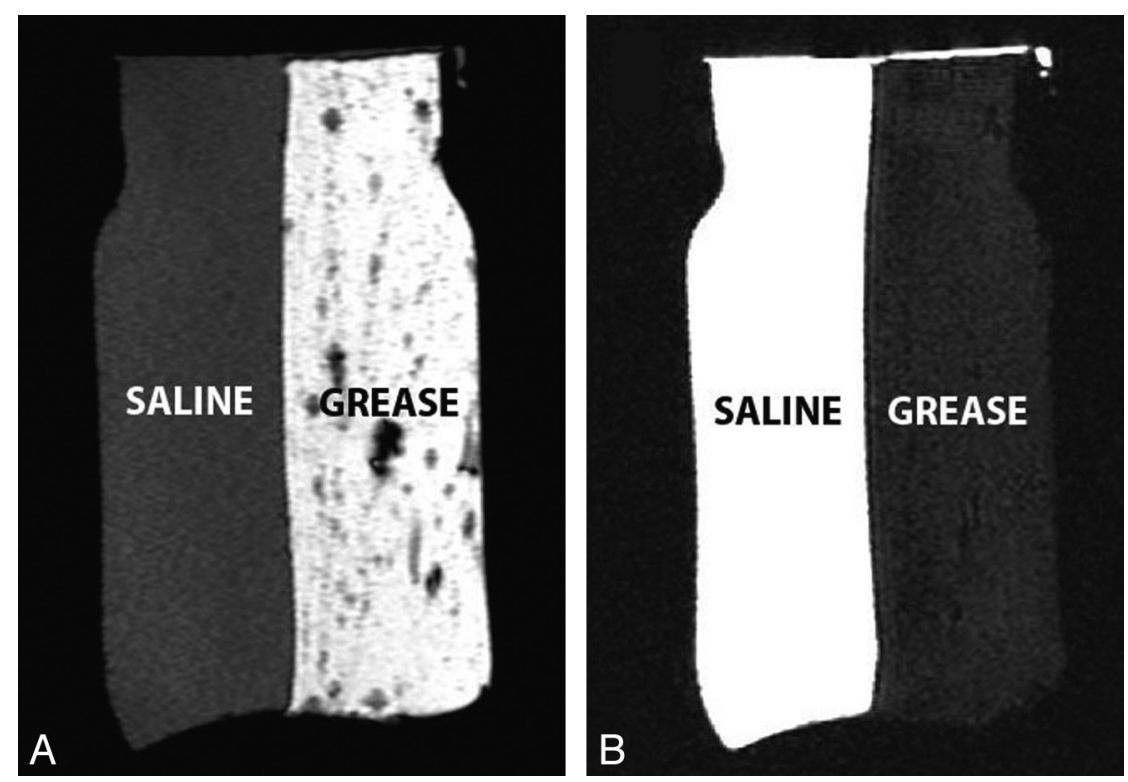

Fig 2. T1- and T2-weighted images of the saline and lubricant grease. $A, T 1$-weighted image reveals hypointensity of the saline and hyperintensity of the lubricant grease. $B$, T2-weighted image reveals hyperintensity of the saline and hypointensity of the lubricant grease

vealed a Hounsfield unit (HU) attenuation of $-100 \mathrm{HU}$, in the range of fat, which is typically $-90 \mathrm{HU}$, rather than fluid, which typically has Hounsfield values of approximately 0 HU (Fig 3). ${ }^{5}$

We are not aware of any previous case reports in which cord compression was caused by an exogenous fluid that entered the epidural space through a blast injury to the thorax. The delayed neurologic symptoms are consistent with previously reported cases of TSEH in which cord compression does not manifest itself until some time ( 7 days to 2.5 months) after the initial injury. ${ }^{6,7}$ In a review of high-pressure injection injuries of a foreign material into the body, the cause of delayed symptoms is described as arising from the combination of the additional volume and evolving inflammatory response because of the presence of a foreign substance. ${ }^{8}$ These factors may help account for the delayed onset of symptoms in our patient.

In conclusion, we report an unusual presentation of a traumatic spinal epidural fluid collection, caused by a high-pressure injection injury of lubricant grease to the upper thorax and into the thoracic spinal canal. The spinal epidural fluid collection mimicked the appearance of an early subacute spinal epidural hematoma on spinal MR imaging. Again, the case of our patient illustrates the importance of detailed clinical information, including a thorough understanding of the mechanism of trauma at the time of interpretation of spi- 


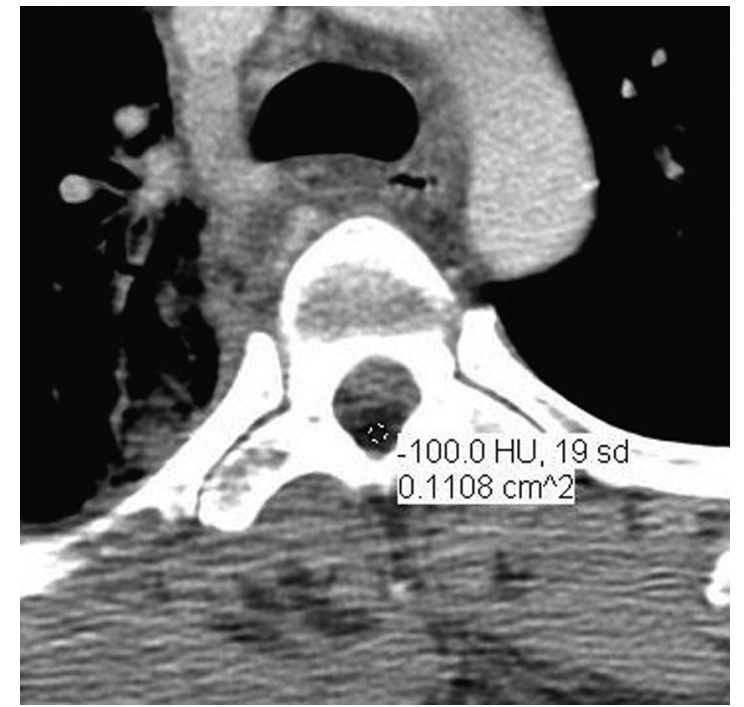

Fig 3. Axial CT revealing evidence of an epidural fluid collection in the thoracic spine displacing the cord anteriorly and to the left. The fluid collection has a attenuation of -100 HU. nal imaging studies, since cord compression can rarely be caused by nonhemorrhagic fluid collection.

\section{Acknowledgment}

We thank Gail Mann, radiology technologist, for her assistance.

\section{References}

1. Harris JH. Mirvis SE. The Radiology of Acute Cervical Spine Trauma. Baltimore: Williams \& Wilkins; 1996:161

2. Bradley WG Jr. MR appearance of hemorrhage in the brain. Radiology 1993:189:15-26

3. ConocoPhillips. Home $>$ Brands and Products $>$ Conoco $>$ Greases. 2009 02/08 [cited 2009 March 26]; Available at: http://www.conocophillipslubricants.com/ documents/conoco/greases/FOB\%20Dynalife\%20GCLB\%20TDS\%20Web.pdf. Accessed September 23, 2009

4. Foo D, Rossier AB. Post-traumatic spinal epidural hematoma. Neurosurgery 1982:11:25-32

5. Huda W. Review of Radiologic Physics. Baltimore: Lippincott Williams \& Wilkins; 2002:122

6. Newey ML, Cain CM. Delayed focal neurological deficit secondary to a cervico-thoracic spinal cord epidural haematoma. Eur Spine J 1999;8:332-33

7. Cuenca PJ, Tulley EB, Devita D, et al. Delayed traumatic spinal epidural hematoma with spontaneous resolution of symptoms. J Emerg Med 2004;27:37-41

8. Hogan CJ, Ruland RT. High-pressure injection injuries to the upper extremity: a review of the literature. J Orthop Trauma 2006:20:503-11 